\title{
Two pathways to suicidal intention in Ecuadorian adolescents: The role of parental and peer attachment, depression and impulsivity
}

\section{Autores}

Nieves Moyano, Katherine Vélez, Andrea Arias, María del Mar Sánchez-Fuentes.

\begin{abstract}
The present study investigated the relationship between attachment patterns -trust, communication and alienation- related to mother, father and peers, depression and impulsivity in suicidal intention among adolescents, by a mediational and moderational analysis. We evaluated the data of 395 Ecuadorian adolescents (54.2\% males, $45.8 \%$ females) aged $14-19$ years $(M=15.86, S D=1.00)$ recruited from several schools. They completed the following measures: the Inventory of Parent and Peer Attachment, the Beck Depression Inventory and the Plutchik Impulsivity Scale. The mediational analyses indicated that, attachment -paternal trustworthiness and alienation from both mother and peers- predicted suicide intention by two pathways: a more direct one mediated by depression; another pathway in which the mediating role of impulsivity was added between attachment and depression. No moderation effects were found. The role of lack of paternal trustworthiness and alienation/negative feelings toward mother and peers are highlighted in the development of depression, which played a mediating role in suicide intention. The role of negative social interactions and negative emotions in relation to suicide intention is discussed, which has several implications for prevention programs for adolescents.
\end{abstract}

Palabras clave

Attachment, Depression, Impulsivity, Suicide intention, Adolescents. 